\title{
Automated Quantitative Assessment of Proteins' Biological Function in Protein Knowledge Bases
}

\author{
Gabriele Mayr, ${ }^{1}$ Günter Lepperdinger, ${ }^{2}$ and Peter Lackner1 \\ ${ }^{1}$ Department of Molecular Biology, University Salzburg, Hellbrunner Strasse 34, 5020 Salzburg, Austria \\ ${ }^{2}$ Institute for Biomedical Aging Research, Austrian Academy of Sciences, Rennweg 10, 6020 Innsbruck, Austria
}

Correspondence should be addressed to Peter Lackner, peter.lackner@sbg.ac.at

Received 29 February 2008; Revised 30 April 2008; Accepted 12 May 2008

Recommended by Rita Casadio

Primary protein sequence data are archived in databases together with information regarding corresponding biological functions. In this respect, UniProt/Swiss-Prot is currently the most comprehensive collection and it is routinely cross-examined when trying to unravel the biological role of hypothetical proteins. Bioscientists frequently extract single entries and further evaluate those on a subjective basis. In lieu of a standardized procedure for scoring the existing knowledge regarding individual proteins, we here report about a computer-assisted method, which we applied to score the present knowledge about any given Swiss-Prot entry. Applying this quantitative score allows the comparison of proteins with respect to their sequence yet highlights the comprehension of functional data. $p f s$ analysis may be also applied for quality control of individual entries or for database management in order to rank entry listings.

Copyright ( 2008 Gabriele Mayr et al. This is an open access article distributed under the Creative Commons Attribution License, which permits unrestricted use, distribution, and reproduction in any medium, provided the original work is properly cited.

\section{Introduction}

Life scientists seek to accumulate knowledge about the distinct functions of biomolecules. Currently, approximately 20000 gene loci with a total of up to 35000 transcripts have been reported for the human genome and transcriptome, respectively $[1,2]$. Genomics and proteomics also greatly support efforts of systems biology, which may certainly provide a better understanding of complex biological processes at the organismic level. The prerequisite for this endeavour is to gain as much knowledge as possible about any functional feature in respect to any particular gene product. Therefore, many disciplines in life sciences use protein sequence data for comparative studies in order to assess distinct functional properties in the context of particular biological situations. Beside printed publications, most data acquired have been collected and assembled into a variety of publicly available databases [3-5]. These are generally accepted to represent a common source of knowledge for research related to biochemistry, molecular biology, biomedicine, or systems biology. In addition, to simply archiving data, interdisciplinary efforts are currently being undertaken to fully annotate genomic and proteomic sequence data sets [6]. The overall goal in this context is to increase the general and detailed understanding about genomes and proteomes.

UniProtKB/Swiss-Prot and UniProtKB/TrEMBL store protein primary data and associated biological and biochemical information. These two are often used as the prime source for data mining. In this study, we focused on the knowledge regarding functional aspects of proteins archived in Swiss-Prot because this repository is generally believed to be a high-quality, manually curated protein knowledge base [7]. Moreover, relevant information is primarily extracted from original publications and review articles, and qualified feedback from external experts is also taken into consideration by the curators of Swiss-Prot. By implementing standardized operational procedures, they build up and edit individual entries [8]. Briefly, Swiss-Prot entries contain information concerning literature references, functional descriptions, domain structure, isoforms, and many more selected information regarding the respective protein [3]. Most entries provide a summary of functional aspects as well as other details, such as posttranslational modifications within their COMMENTS field. Firm data such as those derived from biochemical analyses are assigned clear-cut attributes. For instance, the various properties of an 
enzyme are recorded by a CATALYTIC ACTIVITY as well as by a FUNCTION attribute. Yet, in cases other than enzymes, any other biological function can also be found within the FUNCTION attribute. Biologically, relevant information is also reflected in the protein name (DESCRIPTION line). Other categories such as ENZYME REGULATION, PATHWAY, and TISSUE provide only little further information concerning the protein functional properties than already defined by FUNCTION OR CATALYTIC ACTIVITY.

When using computer-assisted methods, the primary structure of proteins can be easily searched for likely regions of resemblance. Annotated knowledge can be adopted by applying automated bioinformatic routines for those sequences, which are not explicitly listed in Swiss-Prot, but however, share remarkable similarity with experimentally well-studied proteins or protein sequence motifs that have previously been characterized in detail $[7,9,10]$. It has now become generally accepted that information available for proteins from closely-related species can be linked to the human proteome. However, complex information regarding experimental data still has to be manually extracted from published literature or databases. In order to enhance this tedious procedure, we here report a novel method, which weighs the quality of an entry with respect to proposed functional properties of proteins. In order to accomplish that, all textual descriptions within Swiss-Prot were scanned for expressions that specify whether experimental analysis regarding the proteins' biological function is yet in progress or whether published results are still insufficient in order to accurately deduce the protein's role with respect to any particular biological mechanism, pathway, or process. The output of our computerassisted examination resulted in a score for every database entry, which grades the currently existing knowledge and was, therefore, termed the "protein's function score" ( $p f s)$.

In parallel to the development of the $p f s$ procedure, we performed a peer survey. A representative group of trained biologists was asked to manually assess the functional knowledge for 30 randomly selected SwissProt entries. The outcome and feedback guided us when specifically moulding the computer-assisted procedure for the evaluation of currently available information concerning the individual functional properties of proteins. The resulting computational measure was subsequently used for benchmarking of the current knowledge regarding entries in data repositories and libraries. This relation allowed us to incorporate $p f s$ into the result list of protein-protein sequence comparisons. This particular feature provides a reliable indication of how well a particular query hit has been previously annotated regarding functional assignments. Precomputed protein's function scores $(p f s)$ and online BLASTP searches against $p f s$-annotated Swiss-Prot database are accessible at http://biwww.che.sbg.ac.at/PFS.

\section{Methods}

\subsection{Peer Survey}

Database entries were randomly selected. 13 peers comprising of one undergraduate student who has been trained in molecular biology, five $\mathrm{PhD}$ students, five post doctoral fellows, and three principal investigators working as scientists in the field were asked to validate the information provided with these particular entries and to regard the displayed existing knowledge about the protein as high, medium, or low.

\subsection{Computing Knowledge Factor-quantitative Evaluation of Database Entries}

A simple rule was generated in order to yield a solid estimate for the information content of Swiss-Prot: for every single entry, a score was compiled named $p f s$, which is the sum of factors computed for the respective sections of a Swiss-Prot entry, where LITERATURE REFERENCES is denoted $f_{1}$, DESCRIPTION $=f_{d}$, FUNCTION, and CATALYTIC ACTIVITY $=f_{f}$ (Figure 1$)$. At the beginning of the evaluation procedure, value " 1 " was assigned to $f_{1}, f_{d}$, and $f_{f}$ as long as the corresponding section displayed any content. Before summing up all increments, which would yield highest possible value of 3 for $p f s$, all fields were searched for words or phrases of devaluating meanings, which in due course result in the decrease of the relevant factors, $f_{1}, f_{d}$, and $f_{f}$. Terms were selected, which reflect the deliberation of authors to express uncertainty (Table 1). Several of the terms with a specified meaning have been defined by Swiss-Prot [11]. For example, the DESCRIPTION or FUNCTION fields may contain terms which underline the complete or near inability to assign biological function, such as "unknown," "not known," "unnamed," "hypothetical," and "uncharacterized." In cases where the DESCRIPTION field contains the term "putative," $f_{d}$ is decreased to 0.5 . If the DESCRIPTION includes "unnamed," this would result in a zero contribution to $p f s$. In addition to this, a factor was assigned less weight when the entry was qualified as to be deposited "by similarity." During the computational evaluation, each field was split into single sentences and each sentence was searched for devaluation phrases. When analyzing a sentence, the phrase list was searched from top to bottom. The first match determined the reduction of the respective factor $f$. Validation of literature references was processed as follows: firstly, every single literature reference was evaluated with respect to the same criteria as described for $f_{d}$ and $f_{f}$, that is, every single literature reference was searched for keywords, and subsequently, the average raw literature factor was calculated. Exceptionally, Swiss-Prot entries may be the result of automated submissions of genome or expressed sequence tags (ESTs) sequencing data. The accompanying publications rarely address functional questions nor do they experimentally verify bioinformatic analyses via firm biochemical investigations prior to submission to SwissProt. Therefore, Swiss-Prot added a special attribute for large scale experiments, such as genome sequencing or functional genomic approaches. These publication formats were rated zero. Apparently, a large number of publications is often a good reflection of accumulating knowledge. However, $f_{1}(p f s$ increment for literature) should not overwhelm the value of functionally-related terms as defined before. As a matter of 


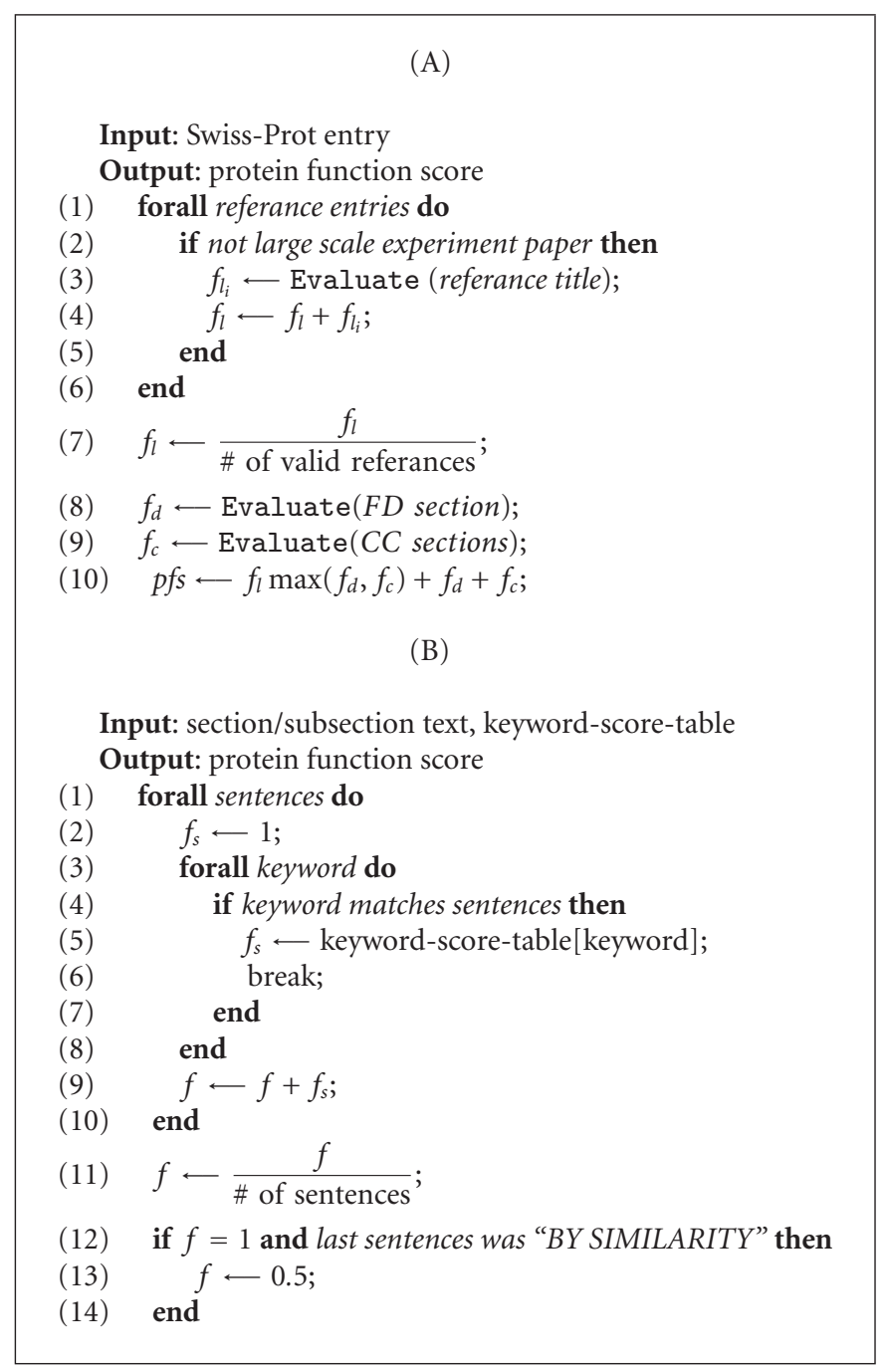

Figure 1: Computation of $p f s$. (A) $p f s$ was calculated with regard to the validity of bibliographic citations as well as a description and comments section. In line 9, the FUNCTION and CATALYTIC ACTIVITY records were evaluated. In step 2, automated entries resulting from large-scale experimental approaches were detached from further analysis. (B) Section evaluation. In every section, every single sentence was evaluated independently. Lines 12-14 are required in case literature citations contain down-weighting phrases, yet conclusions regarding functional properties of a protein have eventually been made by mere resemblance at the primary sequence level (Swiss-Prot term: "BY SIMILARITY").

fact, entries for proteins with largely unknown molecular function typically have $f_{d}$ and $f_{f}$ values that are low or close to zero. In cases of entries that contain numerous publications, the mere addition of an average raw literature factor to the aforementioned low $f_{d}$ and $f_{f}$ would yield a deceptively high $p f s$ value. Therefore, the average raw literature factor was compared to the assessment calculated from the sections DESCRIPTION, FUNCTION, and CATALYTIC ACTIVITY, respectively. The average literature factor was factorized by multiplication with the maximum of $f_{d}$ and $f_{f}$. The proposed scheme has been built and refined iteratively by reading the content of several hundred Swiss-Prot entries and checking the coherence with the calculated $p f s$. The source code for computing pfs for Swiss-Prot is available upon request.

\section{Results}

\subsection{Evaluation of Database Entries by Peers}

Scientists, who routinely use Swiss-Prot or have been trained to extract information about protein from a variety of data repositories, were asked to evaluate 30 randomly picked Swiss-Prot entries. Eventually, the quality of all data as provided within the Swiss-Prot entry for this particular protein should be regarded as being high, medium, or low (Table 2). In cases, where the biological role or the functional property of the respective proteins is properly described by published data, the cumulative knowledge about this protein was considered high. In cases, where there is still experimental data necessary to clarify the biological 
TABLE 1: Expressions and corresponding weights used in pfs calculation: terminology of devaluating meaning frequently used in SwissProt entries, which were applied in (1).

\begin{tabular}{lc}
\hline Unknown & 0.0 \\
Not known & 0.0 \\
Not yet known & 0.0 \\
unnamed & 0.0 \\
Uncharacterized & 0.0 \\
Potential & 0.5 \\
Not clear & 0.5 \\
Not yet clear & 0.5 \\
By similarity & 0.5 \\
Putative & 0.5 \\
Similar to & 0.5 \\
Possible & 0.5 \\
Seems to & 0.5 \\
Thought to & 0.5 \\
Could be & 0.5 \\
Uncertain & 0.5 \\
Potentially & 0.5 \\
Might & 0.5 \\
May & 0.5 \\
Presumably & 0.75 \\
Probably & 0.75 \\
Probable & 0.75 \\
\hline
\end{tabular}

function of the protein or the data are purely descriptive, the information content presented in such entries was ranked medium or low. No further sources of information than those provided by Swiss-Prot had been allowed for this particular evaluation. In a few cases, there appeared only little concordance between peer evaluation results (see Table 2, column "maj"). As a lowest degree of consistence, $38 \%$ was obtained regarding database entries P01827 and Q9N2B6, respectively. Total agreement was achieved again in two cases, Q57910 and P05484. Random scoring would have resulted in $30 \%$ of the entries gaining the lowest possible concordance score $(39 \%)$ and $90 \%$ achieving $<62 \%$ of consensus, which is in strong contrast what was attained by the peer group. The lowest possible as well as the highest concordance score were much lower, $7 \%$ and $50 \%$ respectively. Apparently, there seems to be a bias due to personal appreciation in respect to knowledge archived in biological databases. For instance, PI3 assigned grade "high" to two thirds of the entries, while $\mathrm{Ph} 2$ accounted the same number of entries as containing little knowledge. Additionally, the peers also had to report one field for every particular database entry, which had the highest impact on her/his individual decision. In $35 \%$ of the decisions, the COMMENT-FUNCTION line was considered most important, in $16 \%$, it was the protein's name as provided in the DESCRIPTION line, and in 13\%, the reference list was most appealing for the evaluating peers. Lines such as COMMENTS, FEATURES, COMMENTS-CATALYTIC ACTIVITY, CROSS REFERENCES, or KEYWORDS were chosen only in below $10 \%$ of the cases of the individual decision making processes. Most interesting in this context, peers rarely pointed out that the decision was decisively influenced by literature citations that are embedded in most Swiss-Prot entries. We would furthermore like to note that peers, who applied subjective decision making criteria when validating the currently archived knowledge about a protein's function, did not raise any objections to grade individual entries according to set quality ratings such as low, medium, or high. This strongly suggested to us that any quantitative measure resulting from a standardized procedure, with particular attention paying to biological function information as contained in individual database entries would be also appreciated.

\subsection{Computational Evaluation of Database Entries}

Quality assessment of all Swiss-Prot entries was done by putting weight on to what is currently known about the biological function(s) of an individual protein. The information is abstracted in the respective sections of a SwissProt entry LITERATURE REFERENCES, DESCRIPTION and FUNCTION, and CATALYTIC ACTIVITY. These fields were also highly appreciated by the peers for measuring biological function. Therefore, the text included in these sections was carefully evaluated for particular specifications of down-weighing characterizations as shown in Table 1. Next, the above-mentioned database fields of every single Swiss-Prot entry were examined, and the respective information content was weighed in order to recount and score the currently existing knowledge with regard to the protein's functional properties. As described in the methods section, summing the resulting scores that had been calculated from the database lines equals $p f s$ with a maximum value of 3 (Figure 1). The evaluation scheme acts on the assumption that any field contains standardized textual information. In order to ensure that, the procedure relies not only on parsing of the quantity of text contained in every field, we determined whether there is any correlation between word counts of the individual fields reference title, COMMENTS or DESCRIPTION, and $p f s$. When plotting log (number of words) against $p f s$, only a weak correlation of $r=0.66$ after applying Pearson's formula could be observed (when omitting log scaling, correlation decreases to $r=0.42$ ). In addition to this, we observed a rather broad distribution with respect to word count, which makes it hard to believe that $p f_{s}$ may be deduced by merely counting words. We furthermore analyzed the degree of consent between peers scoring and $p f s$. Taking the entire data set into account, only a week correlation of $r=0.58$ could be revealed. When selecting only those cases that resulted in a concordance score of $>62 \%$, which was half of the entries, a correlation of $r=0.78$ was obtained.

As a next step, $p f s$ was used to classify all Swiss-Prot entries by sorting them into three categories, representing a "low" $(0<1)$, "medium" $(1-2)$, and "high" degree of knowledge (>2) presently available with respect to function and/or biological role (Table 3). Low knowledge results from 
TABle 2: Peer survey. 30 randomly picked Swiss-Prot entries (accession number: AccNum) were evaluated by trained biologists (undergraduate: un; PhD student: Ph; postdoctoral fellows: po; principal investigators: PI). Grades given for the provided information, in particular concerning functional properties as exemplified within an entry were "low" (L), "medium" (M) or "high" (H). The concordance of the ranking assigned by the peers is depicted (maj \%), pfs: protein function score.

\begin{tabular}{|c|c|c|c|c|c|c|c|c|c|c|c|c|c|c|c|}
\hline AccNum & un & $\mathrm{Ph} 1$ & $\mathrm{Ph} 2$ & $\mathrm{Ph} 3$ & $\mathrm{Ph} 4$ & $\mathrm{Ph} 5$ & pol & po2 & po3 & po 4 & PI1 & PI2 & PI3 & maj \% & $\mathrm{pfs}$ \\
\hline Q5RDU9 & $\mathrm{L}$ & $\mathrm{L}$ & M & $\mathrm{L}$ & M & M & M & M & M & $\mathrm{H}$ & M & $\mathrm{L}$ & $\mathrm{H}$ & 54 & 1.5 \\
\hline P0A3D8 & $\mathrm{H}$ & M & $\mathrm{H}$ & M & $\mathrm{H}$ & M & $\mathrm{H}$ & $\mathrm{H}$ & $\mathrm{H}$ & $\mathrm{H}$ & $\mathrm{H}$ & M & $\mathrm{H}$ & 69 & 3 \\
\hline P55863 & $\mathrm{H}$ & M & $\mathrm{H}$ & $M$ & $\mathrm{H}$ & $\mathrm{H}$ & $\mathrm{H}$ & $\mathrm{H}$ & $\mathrm{H}$ & $\mathrm{M}$ & $\mathrm{L}$ & $\mathrm{L}$ & $\mathrm{M}$ & 54 & 2 \\
\hline P01827 & M & $\mathrm{H}$ & $\mathrm{L}$ & M & $\mathrm{L}$ & $\mathrm{H}$ & $\mathrm{L}$ & $\mathrm{H}$ & $\mathrm{H}$ & $\mathrm{L}$ & $\mathrm{L}$ & M & $\mathrm{H}$ & 38 & 2 \\
\hline Q57910 & $\mathrm{L}$ & $\mathrm{L}$ & $\mathrm{L}$ & $\mathrm{L}$ & $\mathrm{L}$ & $\mathrm{L}$ & $\mathrm{L}$ & $\mathrm{L}$ & $\mathrm{L}$ & $\mathrm{L}$ & $\mathrm{L}$ & $\mathrm{L}$ & $\mathrm{L}$ & 100 & 0 \\
\hline Q01684 & M & $\mathrm{L}$ & $\mathrm{H}$ & M & M & $\mathrm{H}$ & $\mathrm{L}$ & M & $\mathrm{H}$ & M & M & $\mathrm{L}$ & M & 54 & 3 \\
\hline Q9BT67 & M & $\mathrm{L}$ & $\mathrm{H}$ & M & $\mathrm{H}$ & M & $\mathrm{L}$ & $\mathrm{H}$ & M & $\mathrm{H}$ & $\mathrm{H}$ & M & $\mathrm{H}$ & 46 & 1,37 \\
\hline P52661 & M & $\mathrm{H}$ & $\mathrm{L}$ & M & M & $\mathrm{H}$ & $\mathrm{H}$ & M & $\mathrm{L}$ & $\mathrm{H}$ & M & $\mathrm{H}$ & $\mathrm{H}$ & 46 & 2,75 \\
\hline Q6GH65 & $\mathrm{L}$ & $\mathrm{L}$ & $\mathrm{L}$ & $\mathrm{L}$ & $\mathrm{L}$ & $\mathrm{L}$ & $\mathrm{L}$ & $\mathrm{L}$ & $\mathrm{L}$ & $\mathrm{M}$ & $\mathrm{L}$ & $\mathrm{L}$ & $\mathrm{M}$ & 85 & 1 \\
\hline Q27459 & $\mathrm{H}$ & $\mathrm{H}$ & M & $\mathrm{H}$ & M & $\mathrm{H}$ & $\mathrm{H}$ & $\mathrm{H}$ & $\mathrm{H}$ & $\mathrm{H}$ & $\mathrm{H}$ & $\mathrm{H}$ & $\mathrm{H}$ & 85 & 3 \\
\hline P0A525 & $\mathrm{L}$ & $\mathrm{L}$ & $\mathrm{L}$ & $\mathrm{L}$ & M & $\mathrm{L}$ & $\mathrm{L}$ & $\mathrm{L}$ & $\mathrm{L}$ & $\mathrm{M}$ & $\mathrm{L}$ & $\mathrm{L}$ & $\mathrm{L}$ & 85 & 0 \\
\hline O77695 & $\mathrm{H}$ & $\mathrm{L}$ & M & M & $\mathrm{H}$ & $\mathrm{L}$ & M & $\mathrm{L}$ & $\mathrm{H}$ & $\mathrm{H}$ & $\mathrm{H}$ & $\mathrm{H}$ & $\mathrm{H}$ & 54 & 3 \\
\hline P05484 & $\mathrm{H}$ & $\mathrm{H}$ & $\mathrm{H}$ & $\mathrm{H}$ & $\mathrm{H}$ & $\mathrm{H}$ & $\mathrm{H}$ & $\mathrm{H}$ & $\mathrm{H}$ & $\mathrm{H}$ & $\mathrm{H}$ & $\mathrm{H}$ & $\mathrm{H}$ & 100 & 3 \\
\hline Q5ZS69 & M & $\mathrm{L}$ & $\mathrm{L}$ & M & M & M & $\mathrm{L}$ & $\mathrm{L}$ & M & M & $\mathrm{L}$ & M & M & 62 & 1 \\
\hline Q8RE08 & $\mathrm{H}$ & $\mathrm{L}$ & $\mathrm{L}$ & M & $\mathrm{H}$ & $\mathrm{H}$ & $\mathrm{L}$ & M & $\mathrm{H}$ & $\mathrm{H}$ & $\mathrm{L}$ & $\mathrm{H}$ & $\mathrm{H}$ & 54 & 2 \\
\hline Q55720 & $\mathrm{M}$ & $\mathrm{L}$ & $\mathrm{L}$ & $\mathrm{L}$ & M & $\mathrm{L}$ & $\mathrm{L}$ & $\mathrm{L}$ & $\mathrm{L}$ & $\mathrm{M}$ & $\mathrm{L}$ & $\mathrm{L}$ & M & 9 & 1 \\
\hline Q9GZP7 & M & $\mathrm{L}$ & $\mathrm{L}$ & M & M & M & M & $\mathrm{H}$ & M & $\mathrm{H}$ & $\mathrm{H}$ & M & $\mathrm{H}$ & 54 & 2,33 \\
\hline P26997 & $\mathrm{H}$ & $\mathrm{H}$ & $\mathrm{H}$ & M & M & $\mathrm{H}$ & $\mathrm{H}$ & $\mathrm{H}$ & M & $\mathrm{H}$ & $\mathrm{H}$ & $\mathrm{H}$ & $\mathrm{H}$ & 77 & 2,75 \\
\hline Q8YFN7 & $\mathrm{L}$ & $\mathrm{L}$ & $\mathrm{L}$ & M & M & M & $\mathrm{H}$ & $\mathrm{L}$ & M & $\mathrm{M}$ & $\mathrm{L}$ & M & $\mathrm{M}$ & 54 & 1,5 \\
\hline P50352 & M & $\mathrm{L}$ & $\mathrm{L}$ & M & M & M & M & M & $\mathrm{H}$ & M & M & M & $\mathrm{H}$ & 69 & 3 \\
\hline Q831V0 & $\mathrm{H}$ & $\mathrm{L}$ & M & $\mathrm{H}$ & $\mathrm{L}$ & $\mathrm{H}$ & $\mathrm{H}$ & $\mathrm{H}$ & $\mathrm{H}$ & M & M & $\mathrm{H}$ & $\mathrm{H}$ & 62 & 2 \\
\hline O67637 & $\mathrm{L}$ & $\mathrm{L}$ & $\mathrm{L}$ & $M$ & $\mathrm{H}$ & $\mathrm{H}$ & $\mathrm{M}$ & $\mathrm{L}$ & M & M & $\mathrm{L}$ & $\mathrm{M}$ & $\mathrm{H}$ & 46 & 1,75 \\
\hline P34180 & $\mathrm{H}$ & M & M & $\mathrm{H}$ & $\mathrm{M}$ & $\mathrm{H}$ & $\mathrm{H}$ & $\mathrm{H}$ & $\mathrm{H}$ & $\mathrm{H}$ & M & $\mathrm{H}$ & $\mathrm{H}$ & 69 & 3 \\
\hline Q16661 & $\mathrm{H}$ & M & $\mathrm{H}$ & $\mathrm{H}$ & $\mathrm{H}$ & $\mathrm{H}$ & $\mathrm{H}$ & $\mathrm{H}$ & $\mathrm{H}$ & $\mathrm{H}$ & $\mathrm{H}$ & $\mathrm{H}$ & $\mathrm{H}$ & 85 & 2,7 \\
\hline O13326 & $\mathrm{H}$ & $\mathrm{H}$ & $\mathrm{L}$ & $\mathrm{H}$ & M & $\mathrm{H}$ & $\mathrm{H}$ & $\mathrm{H}$ & $\mathrm{H}$ & M & $\mathrm{H}$ & $\mathrm{H}$ & $\mathrm{H}$ & 77 & 3 \\
\hline Q9N2B6 & M & $\mathrm{L}$ & $\mathrm{L}$ & M & $\mathrm{L}$ & M & $\mathrm{H}$ & $\mathrm{L}$ & M & $\mathrm{H}$ & $\mathrm{L}$ & M & $\mathrm{H}$ & 38 & 1,75 \\
\hline P84082 & M & M & $\mathrm{L}$ & $\mathrm{H}$ & M & $\mathrm{H}$ & $\mathrm{M}$ & $\mathrm{H}$ & $\mathrm{H}$ & $\mathrm{M}$ & $\mathrm{L}$ & M & $\mathrm{H}$ & 46 & 2,7 \\
\hline Q6GGC5 & M & $\mathrm{L}$ & $\mathrm{L}$ & M & $\mathrm{L}$ & M & $\mathrm{L}$ & $\mathrm{L}$ & M & M & $\mathrm{L}$ & M & $\mathrm{L}$ & 54 & 1 \\
\hline Q3YSL9 & M & $\mathrm{L}$ & $\mathrm{L}$ & M & M & $\mathrm{H}$ & $\mathrm{L}$ & $\mathrm{L}$ & M & $\mathrm{H}$ & M & M & $\mathrm{H}$ & 54 & 1,75 \\
\hline P71238 & $\mathrm{L}$ & $\mathrm{L}$ & $\mathrm{L}$ & $\mathrm{L}$ & M & M & $\mathrm{L}$ & $\mathrm{L}$ & M & $\mathrm{H}$ & $\mathrm{L}$ & $\mathrm{L}$ & $\mathrm{M}$ & 62 & 1 \\
\hline
\end{tabular}

the fact that the number of publications is low and/or the amount of extracted functional information is minute or currently still unavailable. For proteins with moderate knowledge, either many publications or some evidence of a particular biological function is publicly accessible. Solid knowledge with a high $p f_{s}$ is only reflected by welldocumented, reliable information concerning biological function, in particular, clear-cut statements without speculative statements or negative expressions concerning function. The $p f s$ for all Swiss-Prot entries can be obtained online at http://biwww.che.sbg.ac.at/PFS/.

Due to the fact that this novel quality score reflects commonly available knowledge concerning a protein's func- tion, we next appended $p f s$ to the headers of sequence data files, which we subsequently used for sequence comparisons. When performing protein-protein sequence comparisons with the aid of BLASTP as a search algorithm and SwissProt as a data source, now, the result lists displayed not only a generic protein name, but also provided a list with additional notice specifying the functional information content of any particular hit. In this way, the most informative link becomes highlighted, and furthermore, the quality and quantity of functional data, which are available for a set of proteins related to the subject sequence, can be immediately extracted. This service is also freely accessible online at http://biwww.che.sbg.ac.at/PFS/. 
TABLe 3: $p f$ s analysis of Swiss-Prot entries. Absolute numbers of individual entries that were grouped according to computed $p f s$ value are shown for the most prominent species in Swiss-Prot.

\begin{tabular}{|c|c|c|c|c|c|c|}
\hline \multirow[b]{2}{*}{$n$} & \multicolumn{4}{|c|}{$p f_{s}$} & \multicolumn{2}{|r|}{ Species } \\
\hline & 0 & $<1$ & $1-2$ & $>2$ & 3 & \\
\hline 17169 & 880 & 956 & 4973 & 11240 & 5238 & Homo sapiens \\
\hline 13826 & 441 & 480 & 5326 & 8020 & 3545 & Mus musculus \\
\hline 6493 & 1609 & 1650 & 941 & 3902 & 2470 & Saccharomyces cerevisiae \\
\hline 6312 & 123 & 138 & 1710 & 4464 & 2278 & Rattus norvegicus \\
\hline 6065 & 202 & 594 & 2233 & 3238 & 1613 & Arabidopsis thaliana \\
\hline 4402 & 955 & 1024 & 1117 & 2261 & 1661 & Eschericia coli \\
\hline 4272 & 130 & 145 & 2454 & 1673 & 995 & Bos taurus \\
\hline 3072 & 657 & 797 & 1310 & 965 & 609 & Caenorhabditis elegans \\
\hline 2860 & 753 & 760 & 641 & 1459 & 940 & Bacillus subtilis \\
\hline 2612 & 17 & 19 & 580 & 2013 & 1031 & Drosophila melanogaster \\
\hline 2199 & 49 & 56 & 1118 & 1025 & 515 & Xenopus laevis \\
\hline 1935 & 58 & 63 & 1656 & 216 & 107 & Pongo pygmaeus \\
\hline 1837 & 21 & 25 & 597 & 1215 & 652 & Gallus gallus \\
\hline 1774 & 421 & 460 & 1090 & 224 & 136 & Haemophilus influenzae \\
\hline 1652 & 47 & 54 & 900 & 698 & 481 & Salmonella typhimurium \\
\hline 1536 & 36 & 43 & 982 & 511 & 211 & Brachydanio rerio \\
\hline 1420 & 410 & 495 & 770 & 155 & 107 & Mycobacterium tuberculosis \\
\hline 1401 & 6 & 20 & 489 & 892 & 412 & Oryza sativa \\
\hline 1234 & 1 & 2 & 256 & 976 & 586 & Sus scrofa \\
\hline 1226 & 26 & 36 & 744 & 446 & 324 & Pseudomonas aeruginosa \\
\hline 919 & 3 & 11 & 829 & 79 & 51 & Yersinia pestis \\
\hline 283454 & 14317 & 17142 & 165127 & 101185 & 59443 & Total \\
\hline
\end{tabular}

\section{Discussion}

Functional annotations of proteins are collected and provided in databases. Although bioinformatic analyses are regarded to provide unprecedented precision and high performance and are thus being employed to specifically define biological mechanisms at the molecular, genomic, and cellular level, most researchers in life science still (like to) read and survey data collections without applying a standardized validation method. Since knowledge regarding the biological function of proteins is not evenly distributed in databases, and standardized bioinformatic procedures, which would allow individual researchers to specifically qualify information provided in knowledge databases, have not been established yet, which allow individual researchers to specifically qualify information provided in knowledge databases, we developed a novel method. Assessment and cross validation of the current knowledge regarding proteins presented in Swiss-Prot by this linguistic analysis yielded results, which can be hardly achieved by any sort of peer evaluation procedure. In line with this, it is also not surprising that only in selected cases a strong correlation between results of the presented peer data and $p f s$ characterization became apparent. Moreover, assignment of $p f s$ can be easily adjusted to validate other scientific databases, which, for example, accumulate large amounts of textual descriptions or papers. In the context of annotated protein data, $p f s$ can now be employed to determine the knowledge status of large sets of proteins or groups of homologous proteins as well as to tentatively assign potential functions to any proteome.

Considering the impact on daily research, we propose that $p f s$ is appended to protein identifiers within a sequence comparison result list. In this way, nonexperts in a particular field quickly obtain a ranked listing of knowledge distribution when examining the hit list. In addition, tagging protein identifiers with $p f s$ are most helpful for identifying links, which guide one to the most informative database entry. Hence, we regard $p f s$ as an important improvement for accelerating data mining strategies and to providing new avenues for evaluating data derived from genomic and proteomic projects. In the near future, this tool may become an invaluable tool for curators of knowledge databases, primarily to earmark entries which are of minor quality, in order to either eliminate the entry or to improve it during a subsequent update.

\section{Abbreviations}

$P f s: \quad$ Protein's function score

BLASTP: Basic local alignment search tool.

\section{Acknowledgments}

We thank Natalie Sampson (Institute for Biomedical Aging Research), Stephan Reitinger, and Julius Halaschek-Wiener 
(both University of British Columbia at Vancouver) for fruitful discussions and careful reading of the manuscript. This work was supported by Grant P15909-N04 of the Austrian Science Foundation (FWF). Günter Lepperdinger is an APART fellow of the Austrian Academy of Sciences and supported by the Jubilee Fund of the Austrian National Bank (OeNB 12518) and the Austrian Science Fund, FWF (FSP093).

\section{References}

[1] International Human Genome Sequencing Consortium, "Finishing the euchromatic sequence of the human genome," Nature, vol. 431, no. 7011, pp. 931-945, 2004.

[2] T. Imanishi, T. Itoh, Y. Suzuki, et al., "Integrative annotation of 21,037 human genes validated by full-length cDNA clones," PLoS Biology, vol. 2, no. 6, p. e162, 2004.

[3] R. Apweiler, "Functional information in SWISS-PROT: the basis for large-scale characterisation of protein sequences," Briefings in Bioinformatics, vol. 2, no. 1, pp. 9-18, 2001.

[4] E. Camon, M. Magrane, D. Barrell, et al., "The Gene Ontology Annotation (GOA) database: sharing knowledge in Uniprot with Gene Ontology," Nucleic Acids Research, vol. 32, database issue, pp. D262-D266, 2004.

[5] S. Peri, J. D. Navarro, T. Z. Kristiansen, et al., "Human protein reference database as a discovery resource for proteomics," Nucleic Acids Research, vol. 32, database issue, pp. D497-D501, 2004.

[6] B. Boeckmann, M.-C. Blatter, L. Famiglietti, et al., "Protein variety and functional diversity: Swiss-Prot annotation in its biological context," Comptes Rendus Biologies, vol. 328, no. 1011, pp. 882-899, 2005.

[7] R. Apweiler, A. Bairoch, C. H. Wu, et al., "UniProt: the Universal Protein knowledgebase," Nucleic Acids Research, vol. 32, database issue, pp. D115-D119, 2004.

[8] V. L. Junker, R. Apweiler, and A. Bairoch, "Representation of functional information in the SWISS-PROT data bank," Bioinformatics, vol. 15, no. 12, pp. 1066-1067, 1999.

[9] M. L. Riley, T. Schmidt, C. Wagner, H.-W. Mewes, and D. Frishman, "The PEDANT genome database in 2005," Nucleic Acids Research, vol. 33, database issue, pp. D308-D310, 2005.

[10] Y. Zhang, Y. Yin, Y. Chen, et al., "PCAS-a precomputed proteome annotation database resource," BMC Genomics, vol. 4, article 42, pp. 1-7, 2003.

[11] A. Bairoch, R. Apweiler, C. H. Wu, et al., "The Universal Protein Resource (UniProt)," Nucleic Acids Research, vol. 33, database issue, pp. D154-D159, 2005. 

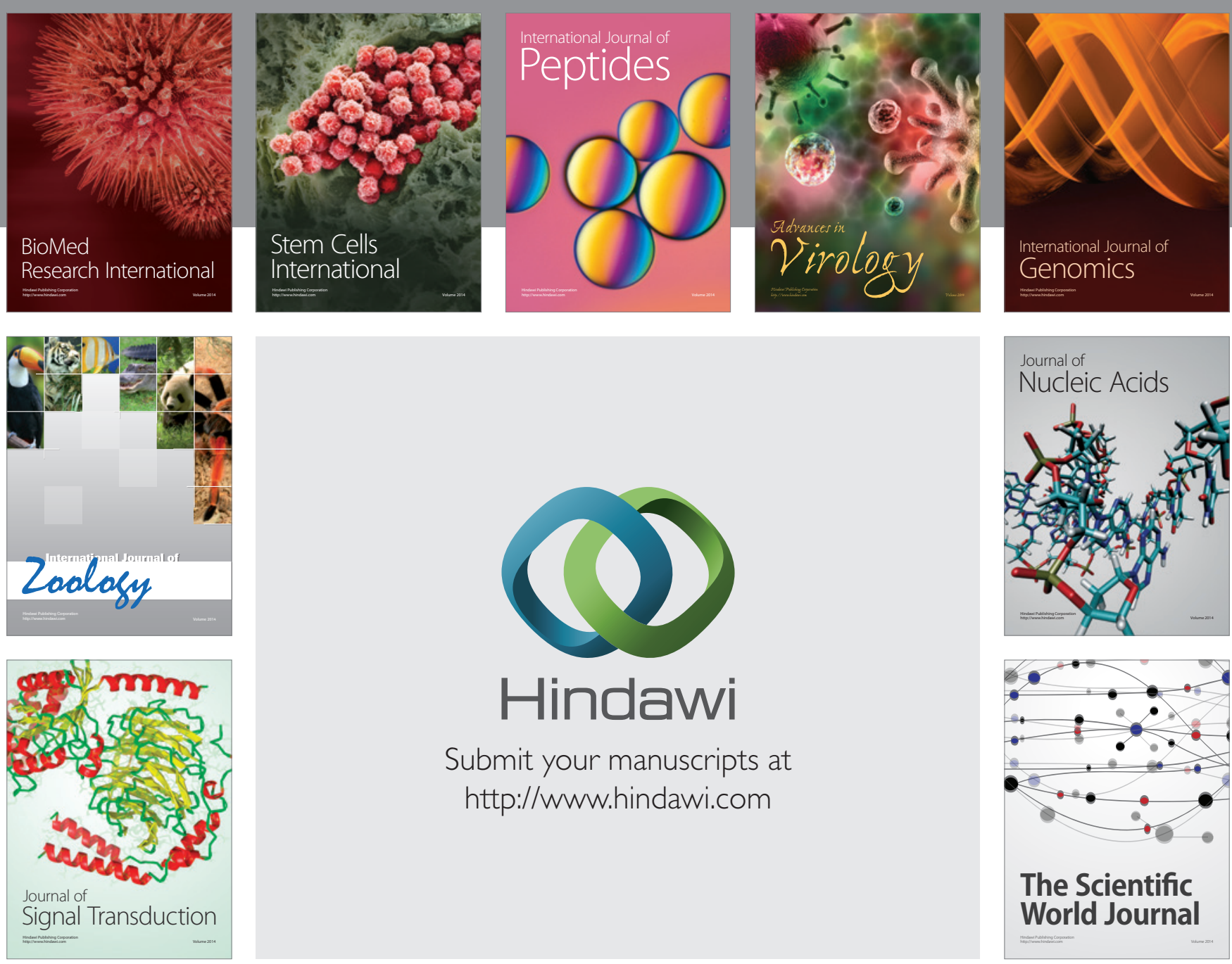

Submit your manuscripts at

http://www.hindawi.com
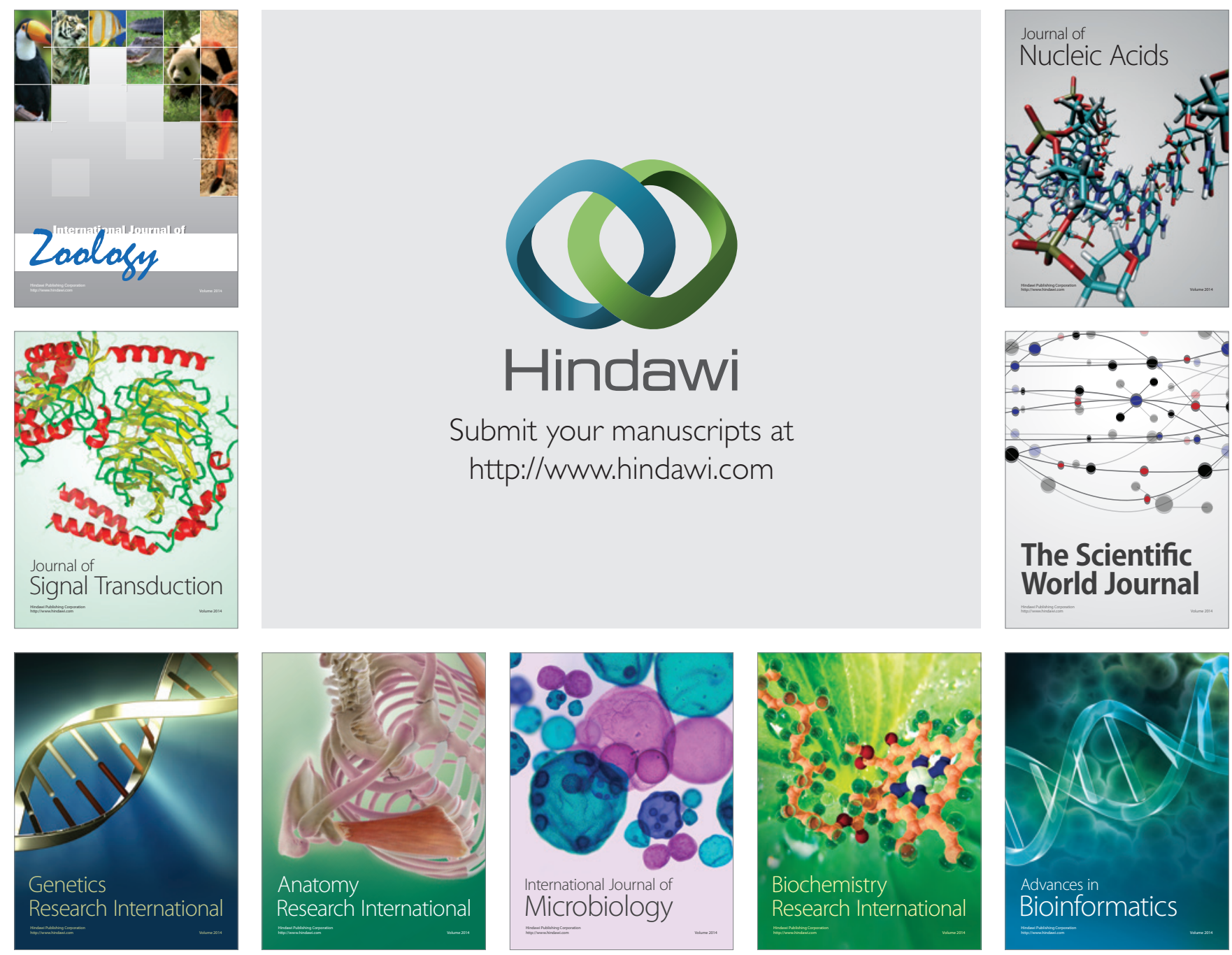

The Scientific World Journal
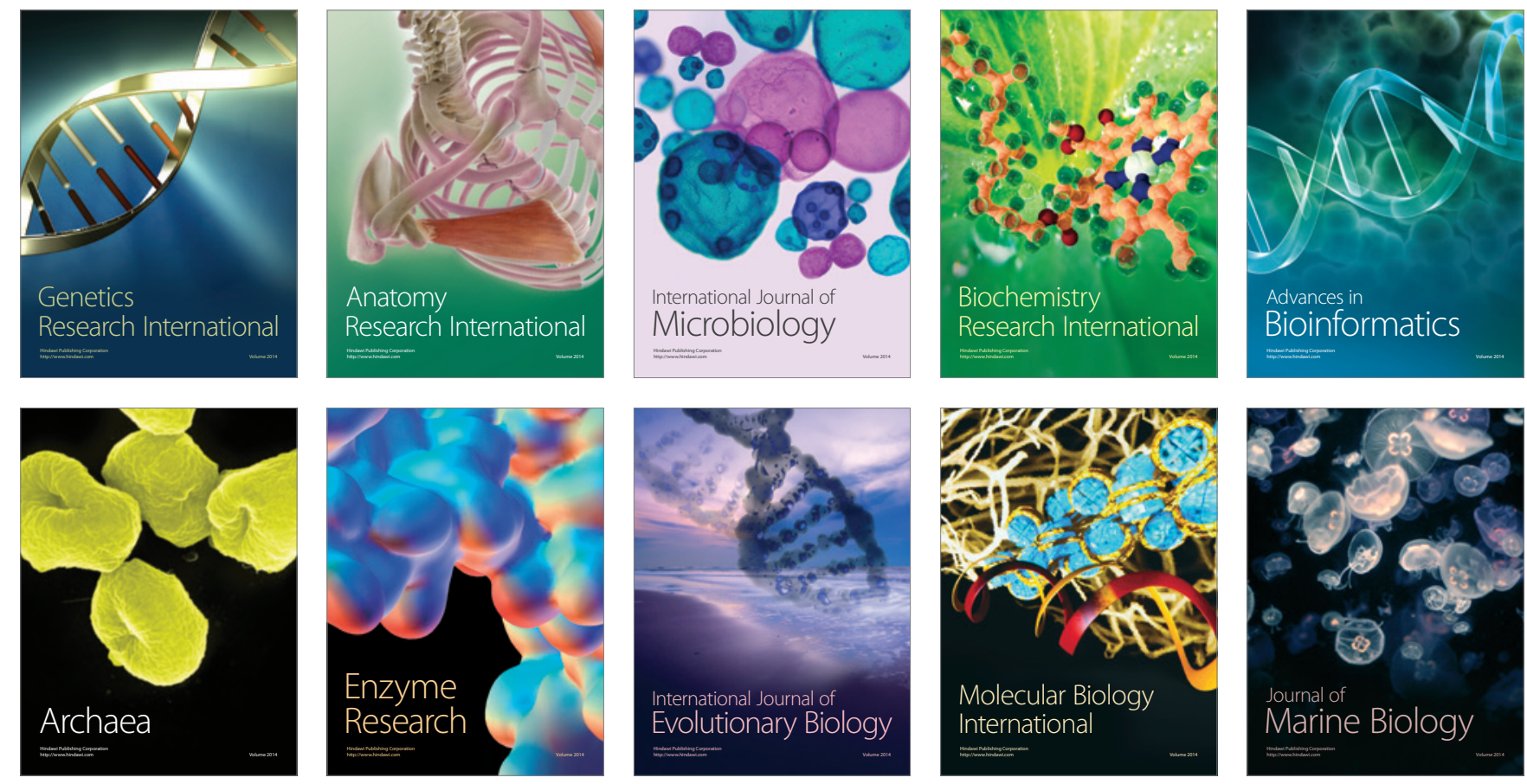